\title{
Optimal Deployment of Charging Stations for Electric Vehicular Networks
}

\author{
Andrea Hess \\ University of Vienna, Austria \\ andrea.hess@univie.ac.at
}

\author{
Claudio Casetti \\ Politecnico di Torino, Italy \\ casetti@polito.it
}

\author{
Francesco Malandrino \\ Politecnico di Torino, Italy \\ malandrino@tlc.polito.it
}

\author{
Moritz B. Reinhardt \\ University of Vienna, Austria \\ moritz.reinhardt@univie.ac.at
}

\begin{abstract}
In a smart city environment, we look at a new, upcoming generation of vehicles running on electric power supplied by on-board batteries. Best recharging options include charging at home, as well as charging at public areas. In this setting, electric vehicles will be informed about public charging stations using wireless communications. As the charging stations are shared resources, cooperating electric vehicles have the potential to avoid unbalanced use of recharging stations and lengthy waiting times.

We present a model for electric vehicles and their battery depletion, vehicle mobility, charging stations, and give a solution for optimal placement of charging stations in a smart city. Our placement approach is based on genetic programming and simulation of electric vehicles which move on a real map of a European city. We show that after a few evolution steps, an optimal solution of the charging infrastructure is derived based on mean trip times of electric vehicles.
\end{abstract}

\section{Categories and Subject Descriptors}

C.2.1 [Computer Communication Networks]: Network Architecture and Design-Wireless communication; I.2 [Artificial Intelligence]: Problem Solving, Control Methods, and Search

\section{Keywords}

Electric Vehicles, Charging Infrastructure Deployment, VANETs, Urban Mobility Modeling

\section{INTRODUCTION}

Despite being at a relatively embryonic stage in terms of market penetration, electric cars represent the most environmentally friendly vehicle as they have absolutely no emissions (unless one accounts for the emission of their primary energy provider, i.e., the power stations). Electric vehicles (e-vehicles) will for sure contribute to overcome the predictable shortage of fossil fuel in the

Permission to make digital or hard copies of all or part of this work for personal or classroom use is granted without fee provided that copies are not made or distributed for profit or commercial advantage and that copies bear this notice and the full citation on the first page. To copy otherwise, to republish, to post on servers or to redistribute to lists, requires prior specific permission and/or a fee.

UrbaNE'12, December 10, 2012, Nice, France.

Copyright 2012 ACM 978-1-4503-1781-8/12/12 ...\$15.00. near future. To support e-vehicles, driving ranges have to be enlarged and electricity suppliers will need to redesign the power grid around new charging stations and schedule their provisioning around the clock in order to offer a high-quality service in terms of usability, low delay, and security.

Vehicle manufacturers are already equipping new vehicles with navigation and infotainment devices as well as with wireless technology to support vehicle to vehicle (V2V) and vehicle to infrastructure (V2I) communication provided by, e.g., 3G and future 4G cellular networks or IEEE $802.11 \mathrm{p}$. Conceivably, this trend will likely continue for e-vehicles. Up-to-date on-board information about the location of a charging station and its status in terms of number of free plugs and average waiting time at the moment is likely to be provided in future vehicles. This information may help individual users to dynamically optimize their travel in terms of reduced search time for charging stations, fares, and overall, reduced trip time. Even governments may leverage this Intelligent Transport Infrastructure (ITS) to foster a decrease of pollution via proper policies (e.g., adapting fares). Additionally, ITS services can inform vehicles about the current road traffic conditions to allow a better choice during peak times. Our final vision is a network of electric vehicles which cooperate to efficiently use the charging infrastructure.

In this paper, we assume the situation where networked electric vehicles are cruising until they detect a low battery charge and, as they are connected via, e.g., a vehicular ad-hoc or cellular network, they receive advertisements of charging stations and navigate to these stations. With today's technology, vehicle charging is still a time-consuming task although fast charging systems capable to charge a battery from 0 to $50 \%$ in a few minutes are being developed $^{1}$. Hence, in a future setting, on demand charging will be one option of a plethora of charging systems, e.g., installed at parking lots, shopping malls, homes, campuses, etc. (We review related work on scenarios of e-vehicles in Section 2.)

In this setting, the main contributions of our paper are:

- We present a model for e-vehicles including state-of-the-art ranges and battery depletion figures and discuss the infrastructure necessary to operate them in an urban environment, i.e., charging stations (Section 3). Further, we introduce a mobility model for on demand charging including a state change (navigation change) for e-vehicles in case of low battery power (Section 4$)$.

- We present a novel approach to optimizing the deployment

\footnotetext{
${ }^{1}$ http://www.betterplace.com
} 
of charging stations using genetic programming. As the deployment of charging stations depends on the mobility of evehicles, which is not observable in real urban areas at higher scales, the real-world behavior is not yet clear. Thus, we selected the genetic programming approach which is capable of adapting to mobility. We describe our approach in Section 5.

- We give first results of a simulation-based study of synthetic vehicular mobility traces on a real city map, i.e., the city map of Vienna, and show that indeed after a few evolution steps, the genetic algorithm produces a solution which is, with statistical certainty, optimal (Section 6).

\section{RELATED WORK}

The integration of electric vehicles into the existing power grid, road traffic, and an ITS infrastructure raises challenging issues that will be discussed in this section.

Recent literature on smart grid systems started paying attention to the integration of vehicles as participants in the electricity market. In [11], e-vehicles are assumed to be consumers and recharge their batteries through the grid, but also conserve capacity. They are expected to react to pricing information when being parked (to delay, reduce, or switch-off charging), and to sell power to the electric grid when feasible (Vehicle to Grid (V2G) power). Although one single vehicle is not providing enough power to sell, vehicle aggregation can save costs as shown in [11]. The V2G concept depends on the power required for moving the vehicles themselves, whether hybrid vehicles are used (carbon and electric power), and on the standing/parking times. In our work, vehicles are integrated as consumers only.

Further, approaches to balance the load on the power grid by regulating charging demand have been investigated. In [4], a congestion pricing model represents driver preferences by the willingness to pay (charging costs and rates). Other concepts for charging scheduling include load shifting dependent on predicted demand [1] or measurement-based grid congestion control [2]. In our approach, we take the perspective of the driver and aim at minimizing the trip time.

Since future urban mobility behavior will be significantly affected by the properties of electric vehicles, mobility models taking the charging demands into account are required. Early simulation studies of e-vehicle mobility account for the energy consumption and charging demands of vehicles during every-day trips. In [23], a mobility model that stochastically determines transitions between daily activities associated with parking locations (workplace, shopping center, etc.), is used to investigate the impact of charging strategies on the energy supply system. When modeling the driving behavior for an information system about nearest charging stations and energy prices, user classes can be differentiated. In [5], users are characterized based on personal information about age, gender, energy market behavior (e.g., minimum accepted charge level), trip type (workday or weekend), and car properties (e.g., battery type). In [19], mobility of e-vehicles is described as being dependent on their need for recharging as well as on the waiting time for charging. The network of charging stations is modeled as a graph and vehicles are attracted by a station and, finally, queue (modeled by queuing networks). Similarly, our approach uses a basic vehicular mobility model and a mode change depending on a battery charge threshold. In our overall concept, the mobility behavior will be further influenced by coooperative decisions about navigation.

To facilitate e-vehicle cooperation, technologies for (ad-hoc) communication between vehicles and with smart grid infrastructures are needed. Recently proposed systems utilize, e.g., cellular network- based text messaging [5] or WMNs [3] for exchanging position, charging state, and available stations nearby, to coordinate charging load in a power grid. In [8], the authors discuss the advantages and disadvantages of different communication technologies available for smart grids, such as wireless mesh networks (WMNs), cellular networks, powerline communication, and digital subscriber lines.

Finally, the improved placement of charging stations has already been targeted in a few studies. For instance, charging stations are placed according to actual energy demands at highway sections as portable storage units [15] or based on day- and nighttime demand estimations for residence and workplace areas of a city [7]. In [21], station topologies are compared in terms of spatial coverage by modeling driver's daily activities as well as e-vehicle adoption (influenced by driver inconvenience in the surrounding). A placement algorithm for charging stations of a door-to-door electric bus service is evaluated in [12] in terms of customer acceptance. The positioning is based on customer distributions extracted from Tokyo taxi traces. In [18], the optimal placement of V2G stations for discharging vehicle batteries onto the grid, to compensate for variabilities of energy generation by renewable sources, is studied.

To get an idea of present charging facilities, we refer the reader to the alternative fueling station locator ${ }^{2}$. This database holds about 4000 electric stations in the US, most of them providing one to four slow and standard charging plugs.

\section{BATTERY CHARGING CHARACTERIS- TICS OF E-VEHICLES}

Users of e-vehicles expect high QoS (Quality of Service) in the delivery of energy given by electricity utility companies. In this context, QoS is defined in terms of how fast a battery can be charged and discharges, energy price, charging delay, and the impact of the charging method on the battery performance. There are several strategies to deliver batteries to e-vehicles [20]: $(i)$ the battery is included in the vehicle price and thus belongs to the vehicle owner, (ii) the battery is owned by a third party that leases them, (iii) the battery is owned by utilities that receive a fixed rate added to the distribution rate. Batteries further differ in terms of charging capability as defined in the SAE J1772 standard $^{3}$ for electrical connectors for e-vehicles:

1. Level 1 (slow charging) implies AC energy to the on-board charger of the vehicle, e.g., $120 \mathrm{~V} / 16 \mathrm{~A}$ for $1.92 \mathrm{~kW}$ charging; charging time $t_{c}: \sim 10 \mathrm{~h}$.

2. Level 2 (standard charging) implies AC energy to the onboard charger of the vehicle, e.g., 208-240V AC, single phase, $12 A-80 A$ for $2.5-19.2 k W ; t_{c}: \sim 6-8 h$.

3. Level 3 (fast charging) implies DC energy from an off-board charger; there is no minimum energy requirement but the maximum current specified is $400 \mathrm{~A}$ and $240 \mathrm{~kW}$ continuous power supplied; $t_{c}: \sim 30 \mathrm{~min}$.

The energy can be supplied by electricity utilities that charge batteries at specific places (home, work, shopping centers, posts in the street, etc.). The daily traffic routine of people plays an important role in the analysis of how batteries are charged and discharged. For example, Zhao et al. [23] show that the locations of parking cars are predominant at work and at home, while shopping only takes place in business hours. It seems clear that Level 1 charging is ideal for

\footnotetext{
${ }^{2}$ http://www.afdc.energy.gov/locator/stations/

${ }^{3}$ published versions of the standard can be purchased here: http://standards.sae.org
} 
charging the vehicle at home (e.g., at night) at low prices. Level 2 charging seems a good solution for charging while the user resides at a place for a long time (e.g., at work), whereas Level 3 seems the solution for fast charging at higher prices when the battery is getting empty and the user does not stop for long. An alternative for recharging batteries is swapping them for charged ones. This solution implies that a charging station is capable of storing a sufficient number of batteries for different vehicle models and of charging them fast. First deployments have recently taken place $^{4}$, however the logistics to implement battery swapping require battery standard agreements between e-vehicle manufacturers [20].

The battery consumption of e-vehicles depends on the intrinsic characteristics of the battery and how the vehicle consumes energy while traveling. For example, let us look at the Nissan Leaf ${ }^{5}$. The Nissan Leaf is powered by a $24 k W h$ lithium ion battery pack. Using an on-board $3.3 \mathrm{~kW}$ charger, the Leaf can be fully recharged from empty in 8 hours from a $220 \mathrm{~V} / 30 \mathrm{~A}$ Level 2 supply. The battery pack is expected to retain $70 \%$ to $80 \%$ of its capacity after 10 years assuming Level 1/2 charging. On the other hand, 80\% of its capacity can be charged in 30 minutes in a Level 3 charger. However, using fast charging as the primary way of recharging, the normal and gradual battery capacity loss is about $10 \%$ higher than regular $220 \mathrm{~V}$ charging over a 10 -year period.

For the Nissan Leaf, the battery lasts for around $160 \mathrm{~km}$ on the EPA (Environmental Protection Agency's) city driving cycle - at $40 \mathrm{~km} / \mathrm{h}$ and with air condition off. However, on highways - at 90 $\mathrm{km} / \mathrm{h}$ and with air condition on - the battery lasts for around 110 $\mathrm{km}$. Considering stop and go traffic in winter - at $25 \mathrm{~km} / \mathrm{h}$ and heater on - the battery lasts for around $100 \mathrm{~km}$ and in heavy stop and go traffic at $10 \mathrm{~km} / \mathrm{h}$ and with air condition on, the battery lasts for around $70 \mathrm{~km}$.

Communications too have an impact on the battery. For example, a DCMA-86P2 using 5.9GHz DSRC protocol supporting communications to vehicle (V2V) or to roadside (V2R) consumes 250 $\mathrm{mW}$ at $6 \mathrm{Mb} / \mathrm{s}$. If a lightweight communication protocol sends 10 packets/s of size $1500 \mathrm{Bytes}$ at $6 \mathrm{Mb} / \mathrm{s}$, the power consumption is approximately in the order of $0.018 \mathrm{kWh}$. For a $24 \mathrm{~kW}$ battery, that consumption represents around $0.075 \%$ of the battery power.

Depletion Model: We use a simple linear depletion model which assumes an average depletion based on the maximum range known from data sheets of manufacturers, e.g., a range of 70$160 \mathrm{~km}$ in an urban area for the Nissan Leaf (100\% capacity, i.e., fully charged battery). The depletion follows the equation

$$
C_{t 2}=C_{t 1}-d \cdot u
$$

where $C_{t 1}, C_{t 2}$ are the capacities of the battery in $\%$ at the two subsequent timesteps $t_{1}$ and $t_{2}, d$ is the distance traveled in $\mathrm{km}$ between $t_{1}$ and $t_{2}$, and $u$ is the average battery use given in $\%$ per $\mathrm{km}$ (where $C_{t 1} \geq d \cdot u$ ). For example, when assuming a range of $100 \mathrm{~km}$ ( $100 \%$ capacity), then $u=1 \%$ per $\mathrm{km}$.

Charging Model: Similar to the depletion model, we use a linear fast charging model (Level 3 charging) of about 30 min charging time (from 0 to $100 \%$ capacity). The charging follows the equation

$$
C_{t 2}=C_{t 1}+\left(t_{2}-t_{1}\right) \cdot r
$$

where $C_{t 1}, C_{t 2}$ are the battery capacities in $\%$ at two subsequent timesteps $t_{1}$ and $t_{2}$, and $r$ is the charging value in $\%$ per min (where

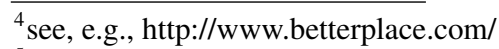

${ }^{5}$ http://en.wikipedia.org/wiki/Nissan_Leaf
}

$\left.C_{t 1}+\left(t_{2}-t_{1}\right) \cdot r \leq 100\right)$. For example, when assuming a charging time of $30 \mathrm{~min}$ (from $0 \%$ to $100 \%$ ), then $r \sim 3.33 \%$ per min.

\section{E-VEHICLE MOBILITY}

Electric vehicular networks show a new type of mobility behavior which is determined by the purpose of a trip, but also by the need to recharge the battery more frequently in a more resourceconstrained environment than by using traditional fuel. Mobility behavior of e-vehicles has been already described in the scope of vehicular ad-hoc networks (VANETs). Vehicular mobility models address different aspects as reflected by the classification provided in [6]: (i) stochastic models select topologies and road characteristics randomly; (ii) traffic stream models look at vehicle flows at a macroscopic level; (iii) car following models account for the relationship between cars and surrounding vehicles in terms of distance, speed, acceleration, etc.; (iv) queueing network models focus on car densities on roads, and $(v)$ behavioral models address driving characteristics derived from social rules. Among the most popular vehicular models are the Nagel-Schreckenberg model [17], the Intelligent Driver Model (IDM) [22], and the Krauss model [14]. The Krauss model provides a safe distance, introduction of a target speed, a jitter to the response to a stimulus, and a stochastic approach to model human irrational behavior (see, e.g., the summary of vehicular mobility models provided in [10]).

\subsection{Extending the SUMO mobility simulator}

A widely-known vehicular traffic simulator is $\mathrm{SUMO}^{6}$ (for further available traffic simulators see $[9,16])$. The SUMO simulator generates mobility based on road networks where movements between source and destination roads are determined by, e.g., a shortest path algorithm. We use and extend the SUMO simulator to generate feasible mobility traces for e-vehicles.

To simulate e-vehicular traffic in a realistic environment, street maps including details about street type, number of lanes, speed limitations, etc. can be imported to SUMO from geodata sources. Figure 1 shows a topology extracted from OpenStreetMap ${ }^{7}$ data of Vienna including traditional fuel gas stations. We re-use actual locations of existing fuel gas stations as an approach to initially position charging stations in an urban environment. However, one caveat is that charging station placement will look different in ten years from now and that different time scales apply for recharging and refueling. The rationale behind using current station positions is their placement in locations preferable w.r.t. demand, reachability, maintaining a minimum distance to the next station, etc.

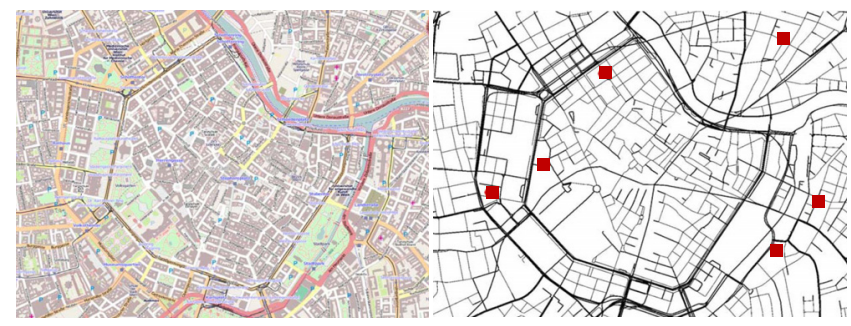

Figure 1: Extract of Vienna's street network imported to SUMO (gas station locations highlighted red).

In addition to positions of charging stations, we extend SUMO by introducing the behavior of e-vehicle charging and use the depletion and charging model for batteries introduced in Section 3.

\footnotetext{
${ }^{6} \mathrm{http}: / /$ sumo.sourceforge.net/

${ }^{7} \mathrm{http}: / /$ www.openstreetmap.org/
} 
Furthermore, the charging model has to assume a possible number of charging plugs provided by a station.

Finally, we assume that the vehicles are equipped with communication as well as positioning and navigation support. While, a priori, vehicles choose a destination based on their trip purpose and use a traditional car following model, we add a route adaptation decision logic which changes the navigation of e-vehicles when recharging is required.

\subsection{Navigating to charging stations on demand}

Whenever charging is needed, e-vehicles change their behavior and try to reach a charging station. This behavior is triggered by a lower threshold termed $T_{l}$. Whenever the State of Charge (SOC) is less than or equal to $T_{l}$, the vehicle changes to follow the attraction model. In the current implementation, the attraction model forces navigating to the charging station closest in distance to avoid running out of battery on the way to the charging station, queuing if necessary, and recharging the battery until an upper threshold $T_{u}$ is reached. Alternatively, an attraction model may minimize overall trip length or trip time. In these cases, care has to be taken to avoid vehicles running out of battery while approaching the selected charging station. After charging, the e-vehicle resumes its purpose-driven navigation (e.g., following the shortest path to a destination). Figure 2 illustrates the state transitions between the basis mobility model and the attraction mobility model.

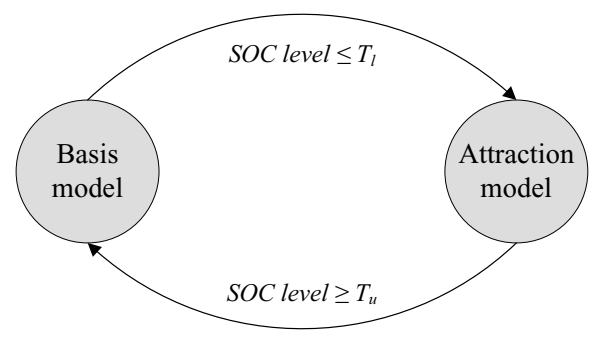

Figure 2: Transition between mobility models triggered by each e-vehicle's level of charging.

Energy supply is another aspect to model. Providing energy supply for each charging station can be either realized by a smart grid or by primary power stations serving a set of nearby stations. To model the sharing of resources between different secondary power stations in the latter case, location and capacity of primary stations have to be taken into account. For our first experiments, we model only the supply distribution between the secondary stations assuming that they are all served by the same primary station.

\section{CHARGING STATION DEPLOYMENT}

As discussed in Section 3, electric vehicles will not have a tremendous range even in the best of conditions, and charging will be a time-consuming task, definitely lengthier than current gas pump stops. It is therefore crucial that the deployment of charging stations be as much as possible in tune with the expected mobility of electric vehicles. It is also important that new variables, specific for electric charging stations, be cast in the problem to be addressed.

We study the problem of charging station deployment through the lenses of optimization and, given a generic city map, we assume that:

- there are $p$ primary power stations each capable of serving up to $X \mathrm{kWh}$;
- charging stations can be deployed in any of $m$ candidate locations;

- each station could be equipped with up to $k$ power plugs.

Notice that, with the above assumptions, each station could host $k$ vehicles in parallel. The amount of power served to such vehicles is limited by the capacity $X$ of its primary station, divided by the number of power plugs currently in use at any of the charging stations served by the same primary station.

Beside the above constraint, it is conceivable that additional constraints can be applied, such as a monetary budget: each candidate location, if selected, drains the budget by some amount. It follows that we can have a limit on the number of both charging stations and the plugs that can be deployed.

As far as the optimization objective is concerned, we select a metric linked to the user satisfaction, namely, the average trip time of electric vehicles. Such a trip time will account for the whole route of the vehicle: from its origin to the selected charging station and from it to the final destination, plus the queuing and recharge times.

\subsection{Deployment Optimization}

Feasible charging station deployments are represented by associating discrete quantities (the number of power plugs to deploy) to the (discrete) elements of a set (the candidate locations). Modeling the deployment through a standard MILP model would result in an overwhelming number of discrete variables, and be computationally infeasible even for small instances of the problem. Indeed, a MILP modelling would not even be feasible unless we derive a closed-form expression for the vehicle trip time (as opposed to simulating the possible solutions). Therefore, we resort to a genetic programming approach [13]. The fitness function, i.e., the objective to minimize, corresponds to the delta of trip time defined earlier. Individual solutions correspond to feasible deployments, i.e., mapping of station IDs in $[0, m]$ to number of plugs in $[0, k]$.

Our algorithm works as follows:

1. the solution pool is initialized with $P$ random solutions;

2. for each solution in the pool, compute its fitness value;

3. use such fitness values to sort the solutions in the pool;

4. discard the bottom fraction $f \cdot P$ of the solution pool, which then has size $(1-f) \cdot P$;

5. pair-wise combine the solutions still in the pool (e.g., selecting all the stations present in both solutions) and obtain $f \cdot P$ new ones;

6. add the newly-constructed solutions to the pool;

7. go to Step 2.

The whole process is repeated for the target number $G$ of generations. The outcome of the process is a set of feasible solutions, along with their fitness values. The best among such solution is, with statistical certainty [13], very close to the actual optimal solution.

Along with its simplicity, a major advantage of genetic programming is that we can tune its behavior by setting the parameters $P$, $G, f$. For example, a larger solution pool (i.e., a higher $P$ ) puts more emphasis on the exploration phase, i.e., trying many different potential solutions. On the other hand, increasing the number $G$ of generations puts more emphasis on the exploitation phase, i.e., improving the existing solutions as much as possible. Given the specific scenario that we take into account, we need to take a balanced approach, and therefore set the parameters as shown in Table 1. 


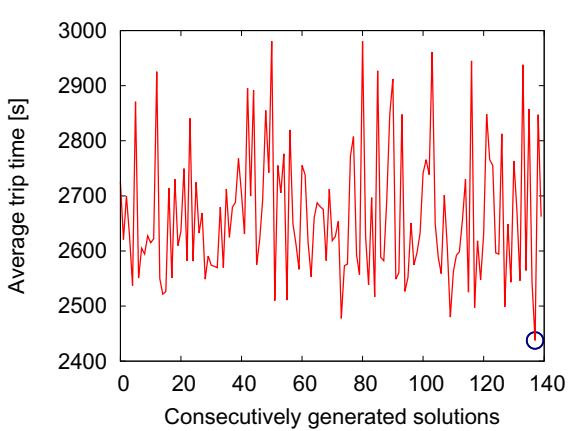

(a)

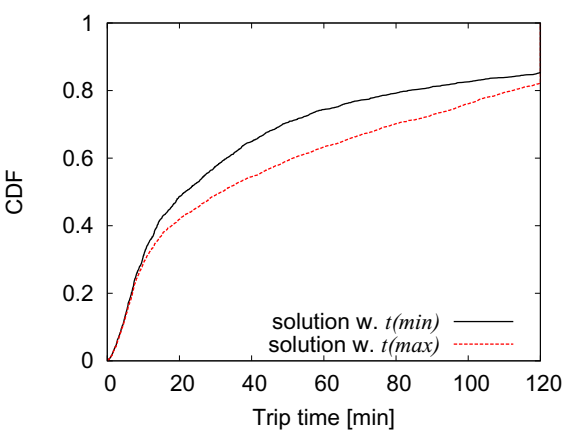

(b)

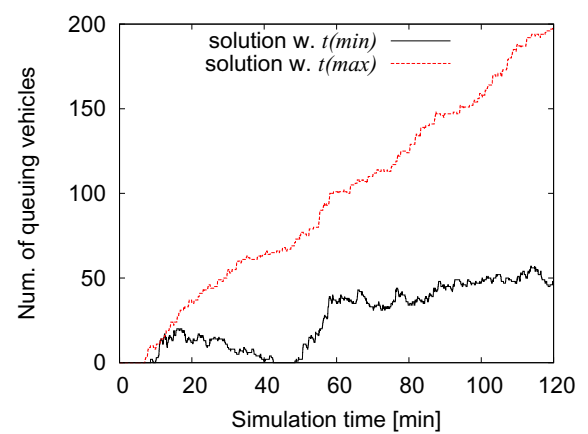

(c)

Figure 3: (a) Average trip time $t$ for the deployment solutions generated by the optimizer. (b-c) Comparison of the solutions with minimum and maximum $t$ in terms of (b) cumulative density functions (CDFs) for trip time and (c) number of vehicles in charging station queues.

\begin{tabular}{r|l} 
Traffic model & \\
Simulation time & $7200 \mathrm{~s}$ \\
Area size & $\sim 36 \mathrm{~km}^{2}$ \\
Number of e-vehicles & 2160 \\
Car following model & Krauss model \\
\hline Depletion model & \\
Battery capacity & $24 \mathrm{kWh}$ \\
E-vehicle driving range & $100 \mathrm{~km}$ \\
Energy consumption per km $u$ & $1 \%$ \\
Lower SOC threshold $T_{l}$ & $5 \%$ \\
Upper SOC threshold $T_{u}$ & $20 \%$ \\
\hline Charging model & \\
Number of charging stations $m$ & 6 \\
Plugs per station $k$ & $0,3,6,9$, or 12 \\
Number of primary stations $p$ & 1 \\
Maximum total capacity & 30 plugs \\
Charging speed $r$ & $3.33 \%$ per min \\
Waiting queue length & infinite \\
Genetic algorithm & \\
Solution pool size $P$ & 40 \\
Discard fraction $f$ & 0.5 \\
Number of generations $G$ & 5
\end{tabular}

Table 1: Simulation parameters.

\section{EXPERIMENTS}

During each experiment, the optimizer iteratively inputs possible charging station configurations to the traffic simulation to compute the corresponding fitness value. In order to account for both power capacity limits and budget constraints, the total number of charging plugs that can be served is set to 30 . The vehicular traffic is simulated on the city center topology of Vienna (Figure 1), where 720 vehicles are initially placed and additional 720 are inserted every hour (one every $5 \mathrm{~s}$ of the remaining simulation time) to keep the traffic density on a constant level. Source/destination locations are randomly selected (uniform distribution). An upper battery level threshold of $20 \%$ is used since $20 \mathrm{~km}$ range can be considered as adequate for the area size of $6 \times 6 \mathrm{~km}$. Besides, this reduces the impact of charging duration on trip time as the optimization should depend on navigation and queuing time, and additionally, allows emulating more charging services in shorter simulation time. As car following model we selected the Krauss model since it provides basic vehicular behavior as described in Section 4 and a robust im- plementation of the Krauss model exists already in SUMO. Table 1 summarizes the parameter setting used for traffic, depletion, charging, and optimization model setup.

We now discuss the results of the optimization process. Figure 3(a) shows the average trip time, i.e., the fitness value, for the solutions generated by the optimizer, as described in Section 5.1. The solution pool is initialized with $P=40$ randomly generated solutions. Then, for each of the $G=5$ subsequent generations, the worst $P \cdot f=20$ solutions are discarded and replaced with new ones, coming from the combination of the others. The whole process takes $P+G \cdot f \cdot P=140$ possible solutions into account. The optimal solution (corresponding to an average trip time of $2438 \mathrm{~s}$, identified by a circle in the plot) is found in the last generation. Here, three charging stations are active with 9 to 12 plugs, whereas in the solution with the largest trip time $(t=2981 \mathrm{~s})$ all the available plugs are activated on one station only. Notice that, unless we test all possible solutions, we cannot exclude that by letting the algorithm run longer, i.e., for more generations, we could find a better one.

To show how the impact varies according to different placement and configuration of charging stations, we compare two metrics for the best and the worst solutions. First, Figure 3(b) shows the cumulative density functions (CDFs) of the observed trip times for the solutions achieving best and worst fitness. While the same fraction of trips have a duration shorter than $10 \mathrm{~min}$ (i.e., recharging might not be necessary), the solution minimizing the trip time rises more steeply between about 10 and 50 min. Further, 3\% less vehicles have here a trip time larger than $120 \mathrm{~min}$. Second, Figure 3(c) shows the number of vehicles in queues for the same example solutions over the simulation time. The queue length values show that the capacity limit of 30 plugs even causes queuing times in the optimal solution. However, the charging demands of our simulation setting can be handled in this case on a more acceptable level (26 vehicles are waiting on average), while the worst solution exhibits an increasing congestion (198 vehicles are waiting after $120 \mathrm{~min}$ ).

\section{CONCLUSION}

We reviewed some critical issues in electric vehicular networks due to battery depletion and charging. Hereby, we identified mobility modeling and charging station deployment as two of the most critical tasks for the widespread adoption of electric vehicles. We assumed the likely situation, that electric vehicle drivers resort to information about charging stations and, in future, to cooperative 
navigation (based on message exchange) to decrease waiting- and trip times.

We characterized the frequency and duration of the battery charging process and proposed a charging model. Further, we introduced a mobility model that includes a state change between general vehicular mobility and navigation to a charging station on demand. To show the feasibility of our approach, we integrated our models into a widely-used traffic simulator. Finally, a genetic programming approach has been employed, which finds a virtually-optimal charging station deployment so as to achieve the minimum possible trip times.

\section{Acknowledgment}

The work described in this paper was partially supported by the EC's FP7 Network of Excellence Euro-NF SJRP e-VENETs. Further, this research was supported by a Marie Curie IEF within the EC's FP7 (contract PIEF-GA-2010-276336 MOVE-R).

\section{REFERENCES}

[1] C. Ahn, C.-T. Li, and H. Peng. Optimal Decentralized Charging Control Algorithm for Electrified Vehicles Connected to Smart Grid. Journal of Power Sources, 196(23):10369 - 10379, 2011.

[2] O. Ardakanian, C. Rosenberg, and S. Keshav. Real-time Distributed Congestion Control for Electrical Vehicle Charging. In ACM SIGMETRICS Greenmetrics Workshop, 2012.

[3] M. Erol-Kantarci, J. Sarker, and H. Mouftah. Communication-based Plug-In Hybrid Electrical Vehicle Load Management in the Smart Grid. In IEEE Symposium on Computers and Communications, 2011.

[4] Z. Fan. Distributed Charging of PHEVs in a Smart Grid. In IEEE International Conference on Smart Grid Communications (SmartGridComm), 2011.

[5] J. Ferreira, P. Pereira, P. Filipe, and J. Afonso. Recommender System for Drivers of Electric Vehicles. In International Conference on Electronics Computer Technology (ICECT), 2011.

[6] M. Fiore. Vehicular Mobility and Network Simulation. Handbook on Vehicular Networks, S. Olariu and M. C. Weigle Eds., Taylor and Francis, 2008.

[7] I. Frade, A. Ribeiro, G. Goncalves, and A. Antunes. Optimal Location of Charging Stations for Electric Vehicles in a Neighborhood in Lisbon, Portugal. Transportation Research Record, 2252:91-98, 2011.

[8] V. Gungor, D. Sahin, T. Kocak, S. Ergut, C. Buccella, C. Cecati, and G. Hancke. Smart Grid Technologies: Communication Technologies and Standards. IEEE Transactions on Industrial Informatics, 7(4):529-539, 2011.

[9] J. Härri, F. Filali, and C. Bonnet. Mobility Models for Vehicular Ad Hoc Networks: A Survey and Taxonomy. IEEE Communications Surveys \& Tutorials, 11(4):19-41, 2009.
[10] H. Hartenstein and K. Laberteaux, editors. VANET Vehicular Applications and Inter-Networking Technologies. John Wiley and Sons, 2010.

[11] S. Kamboj, W. Kempton, and K. S. Decker. Deploying Power Grid-integrated Electric Vehicles as a Multi-agent System. In International Conference on Autonomous Agents and Multiagent Systems (AAMAS), 2011.

[12] H. Kameda and N. Mukai. Optimization of Charging Station Placement by Using Taxi Probe Data for On-Demand Electrical Bus System. In International Conference on Knowledge-Based and Intelligent Information and Engineering Systems (KES), 2011.

[13] J. Koza and R. Poli. Genetic Programming. In E. K. Burke and G. Kendall, editors, Search Methodologies, pages 127-164. Springer US, 2005.

[14] S. Krauß. Microscopic Modeling of Traffic Flow: Investigation of Collision Free Vehicle Dynamics. $\mathrm{PhD}$ thesis, University of Cologne, 1998. http://e-archive.informatik.uni-koeln.de/319/.

[15] Z. Li, Z. Sahinoglu, Z. Tao, and K. H. Teo. Electric Vehicles Network with Nomadic Portable Charging Stations. In IEEE Vehicular Technology Conference Fall (VTC-Fall), 2010.

[16] F. J. Martinez, C. K. Toh, J.-C. Cano, C. T. Calafate, and P. Manzoni. A Survey and Comparative Study of Simulators for Vehicular Ad Hoc Networks. Wireless Communications and Mobile Computing, 11:813-828, 2011.

[17] K. Nagel and M. Schreckenberg. A Cellular Automaton Model for Freeway Traffic. Journal de Physic, I(2):2221-2229, 1992.

[18] F. Pan, R. Bent, A. Berscheid, and D. Izraelevitz. Locating PHEV Exchange Stations in V2G. In IEEE International Conference on Smart Grid Communications (SmartGridComm), 2010.

[19] H. Qin and W. Zhang. Charging Scheduling with Minimal Waiting in a Network of Electric Vehicles and Charging Stations. In ACM International Workshop on Vehicular Inter-networking (VANET'11), 2011.

[20] A. Senart, S. Kurth, and G. Le Roux. Assessment Framework of Plug-in Electric Vehicles Strategies. In IEEE International Conference on Smart Grid Communications (SmartGridComm), 2010.

[21] T. Sweda and D. Klabjan. An Agent-based Decision Support System for Electric Vehicle Charging Infrastructure Deployment. In IEEE Vehicle Power and Propulsion Conference (VPPC), 2011.

[22] M. Trieber, A. Hennecke, and D. Helbing. Congested Traffic States in Empirical Observations and Microscopic Simulations. Physical Review E, 62(2):1805-1824, 2000.

[23] L. Zhao, P. Awater, A. Schafer, C. Breuer, and A. Moser. Scenario-based Evaluation on the Impacts of Electric Vehicle on the Municipal Energy Supply Systems. In IEEE Power and Energy Society General Meeting, 2011. 